\title{
Gating of Sensory Input by Spontaneous Cortical Activity
}

\author{
Artur Luczak, ${ }^{1,2}$ Peter Bartho, ${ }^{1,3}$ and Kenneth D. Harris ${ }^{1,4}$ \\ ${ }^{1}$ Center for Molecular and Behavioral Neuroscience, Rutgers University, Newark, New Jersey 07102, ${ }^{2}$ Canadian Centre for Behavioural Neuroscience, \\ University of Lethbridge, Lethbridge, Alberta, Canada T1K 3M4, ${ }^{3}$ Institute of Experimental Medicine, Hungarian Academy of Sciences, Budapest 1083, \\ Hungary, and ${ }^{4}$ Departments of Bioengineering, Electrical and Electronic Engineering, Imperial College London, London SW7 2AZ, United Kingdom
}

The activity of neural populations is determined not only by sensory inputs but also by internally generated patterns. During quiet wakefulness, the brain produces spontaneous firing events that can spread over large areas of cortex and have been suggested to underlie processes such as memory recall and consolidation. Here we demonstrate a different role for spontaneous activity in sensory cortex: gating of sensory inputs. We show that population activity in rat auditory cortex is composed of transient 50-100 ms packets of spiking activity that occur irregularly during silence and sustained tone stimuli, but reliably at tone onset. Population activity within these packets had broadly consistent spatiotemporal structure, but the rate and also precise relative timing of action potentials varied between stimuli. Packet frequency varied with cortical state, with desynchronized state activity consistent with superposition of multiple overlapping packets. We suggest that such packets reflect the sporadic opening of a "gate" that allows auditory cortex to broadcast a representation of external sounds to other brain regions.

\section{Introduction}

Neuronal activity is not strictly determined by external stimuli, but also exhibits complex spontaneously generated patterns. Spontaneous activity in sensory cortex often resembles sensory responses (Hoffman and McNaughton, 2002; Kenet et al., 2003; Ji and Wilson, 2007; Han et al., 2008) and has been suggested to represent recall of modality-specific components of complex memories triggered by input from higher order structures (Hoffman et al., 2007; O'Neill et al., 2010; Battaglia et al., 2011; Carr et al., 2011). Nevertheless, a unique role for spontaneous activity in memory consolidation is questioned by the similarity of spontaneous activity to activity evoked by previously unexperienced stimuli (Luczak et al., 2009; Dragoi and Tonegawa, 2011).

Spontaneous and evoked neocortical firing patterns share a common fine temporal structure. In sensory systems, the onset of a stimulus evokes a transient but coherent increase in the firing rate of a large number of nearby neurons that we will term an "activity packet." Although the latency and duration of such packets can vary between areas and layers, single-neuron and population recordings indicate that sensory-evoked transients have a typical duration of 50-100 ms (Richmond et al., 1987; deCharms et al., 1998; de Kock et al., 2007; Qin et al., 2007; Benucci et al., 2009; Luczak et al., 2009). Similar packets are

\footnotetext{
Received June 19, 2012; revised Nov. 3, 2012; accepted Nov. 16, 2012.

Author contributions: A.L. and K.D.H. designed research; A.L. and P.B. performed research; A.L. analyzed data; A.L. and K.D.H. wrote the paper.

This work was supported by NIH Grants MH073245 and DC009947 and National Science Foundation Grant SBE0542013 (to the Temporal Dynamics of Learning Center, an NSF Science of Learning (enter) to K.D.H., by Alberta Heritage Foundation for Medical Research and Natural Sciences and Engineering Research Council of Canada grants to A.L., and by the Hungarian Scientific Research Fund (OTKA NF 101773). We thank M. Carandini and M. Okun for comments on a previous version of the manuscript.

The authors declare no competing financial interests.

Correspondence should be addressed to either Artur Luczak at the above address, E-mail: luczak@uleth.ca; or Kenneth D. Harris at his present address: Institute of Neurology and Department of Neuroscience, Physiology, and Pharmacology, UCL, 21 University Street, London WC1E 6DE, United Kingdom. E-mail: kenneth.harris@ucl.ac.uk. DOI:10.1523/JNEUROSCI.2928-12.2013

Copyright $\odot 2013$ the authors $\quad 0270-6474 / 13 / 331684-12 \$ 15.00 / 0$
}

evoked by active sensory movements such as sniffs, whisks, and saccades (Melloni et al., 2009; Shusterman et al., 2011). It is well established that spike timing relative to stimulus onset can encode information (Richmond et al., 1990; Panzeri et al., 2001; Reich et al., 2001; Nelken et al., 2005). Nevertheless, population timing patterns evoked by different stimuli consist of variations on a common sequential structure, with smaller differences in the spike timing of one cell between stimuli than between different cells' responses to one stimulus (Luczak et al., 2009). The onset of spontaneous population firing events show a similar temporal fine structure to sensory onset responses (Luczak et al., 2009), also consistent with intracellular recordings indicating that both onset-evoked and spontaneous activity in quiescent animals are dominated by fluctuations or "bumps" of similar time course (DeWeese and Zador, 2006; Poulet and Petersen, 2008). Nevertheless, spontaneous and evoked firing show different propagation between layers, consistent with their initiation by top-down feedback and thalamic input, respectively (Sakata and Harris, 2009; Takeuchi et al., 2011).

Here we characterize the role of spontaneous activity in auditory cortex of unanesthetized rats listening to extended pure tone stimuli. Tone onsets reliably evoked sequential activity packets of duration 50-100 ms. During the sustained period, population activity was not continuous, but composed of sporadic activity packets grossly similar to those seen during silence. The rate at which these packets occurred varied with cortical state, with desynchronized activity consistent with superposition of multiple overlapping packets. Both the firing rates and precise timing of neurons inside these packets varied with ongoing tone frequency, suggesting that spontaneous activity patterns "gate" the auditory cortical representation of external sounds.

\section{Materials and Methods}

Surgery and recording. All experiments were performed in accordance with protocols approved by the Rutgers University Animal Care and Use Committee. Experimental procedures have been previously described 
(Luczak et al., 2009). Briefly, head post was implanted on the skull of each of four male Sprague Dawley rats (300-500 g) under ketamine-xylazine anesthesia, and a well drilled above the auditory cortex and covered with wax and dental acrylic. After recovery, each animal was trained for $6-8 \mathrm{~d}$ to remain motionless in the restraining apparatus for increasing periods (target, 1-2 h). On the day of the surgery, each animal was briefly anesthetized with isoflurane and the dura resected; after a $1 \mathrm{~h}$ recovery period, recording began. In all experiments, animals stayed motionless for at least $1 \mathrm{~h}$, which was also indicated by stable, clusterable recorded units. Animals were judged to be in a quiescent awake state during this time by the occurrence of spontaneous movements such as postural adjustments and whisking. The location of the recording sites was estimated to be primary auditory cortex by stereotaxic coordinates and vascular structure (Sally and Kelly, 1988; Doron et al., 2002; Rutkowski et al., 2003). Electrodes were estimated to be in deep layers by field potential reversal, and most likely in layer $\mathrm{V}$ due to electrode depth and the presence of broadly tuned units of high background rate (Sakata and Harris, 2009). For recording we used silicon microelectrodes (Neuronexus Technologies) consisting of eight or four shanks spaced by $200 \mu \mathrm{m}$, with a tetrode recording configuration on each shank ( $25 \mu \mathrm{m}$ spacing between sites; typical yield, $5-15$ well-isolated units per tetrode). Units were isolated by a semiautomatic algorithm (http://klustakwik.sourceforge.net) followed by manual clustering (http://klusters.sourceforge.net). Only neurons with firing rates higher than $1 \mathrm{~Hz}$ were used in further analysis, resulting in population sizes $17,26,32$, and 45 for the four rats, respectively. After spike sorting, the activity of all sorted neurons was recombined to produce a multiunit activity (MUA) trace.

In our experiments, we observed that awake animals can also show spontaneous fluctuations in cortical activity that are prominent although smaller than those observed during slow-wave sleep. This is consistent with several other recent studies using multiple modalities including intracellular recording, voltage-sensitive dye imaging, and local field potential (LFP) and population recording (Crochet and Petersen, 2006; DeWeese and Zador, 2006; Ferezou et al., 2006; Luczak et al., 2007; Greenberg et al., 2008; Poulet and Petersen, 2008; Luczak et al., 2009; Sakata and Harris, 2009; Okun et al., 2010). We note that the rats in the present study, as in previous experiments, had been briefly anesthetized with isofluorane $1 \mathrm{~h}$ or more before being placed in a familiar headfixation apparatus where recording took place. Nevertheless, it seems unlikely that residual anesthesia is the cause of the spontaneous fluctuations we observed. Indeed, in previous studies of mice that had woken from isofluorane anesthesia $2-3 \mathrm{~h}$ before recordings, spontaneous fluctuations were instantly abolished when the animal began whisking, returning when whisking stopped (Crochet and Petersen, 2006; Poulet and Petersen, 2008); a different study of rats that woke from isofluorane anesthesia into a previously unexperienced head-fixation condition, only showed desynchronized activity (Constantinople and Bruno, 2011), which may reflect a state of heightened alertness in these animals; in an additional study, periods of population silence ("down states") were found in awake rats, using chronic recordings made more than a week after anesthesia (Vyazovskiy et al., 2011). Together with inferences from low-frequency LFP power in primates (see Discussion) and rodents (Buzsáki et al., 1988; Niell and Stryker, 2010), these studies suggest that the degree of spontaneous fluctuation in cortical population activity changes rapidly with behavioral and cognitive state.

Acoustic stimuli. As stimuli we used pure tones (3.3, 5.4, 8.9, 15, and $24 \mathrm{kHz}$ at $60 \mathrm{~dB}$ ), interleaved with $1 \mathrm{~s}$ periods of silence. All stimuli were tapered at beginning and end with a $5 \mathrm{~ms}$ cosine window. Experiments took place in single-walled sound isolation chamber (IAC) with sounds presented free field (RP2/ES1; Tucker-Davis Technologies). To compensate for the transfer function of the acoustic chamber, tone amplitudes were calibrated before the experiment using a condenser microphone placed next the animal's head (7017; ACO Pacific) and an MA3 microphone amplifier (Tucker-Davis Technologies). Spontaneous activity was recorded for $10 \mathrm{~min}$ before and after presenting stimuli. Additionally, during stimulus presentation, activity occurring $>200 \mathrm{~ms}$ after stimulus offset and before the next stimulus onset was also regarded as spontaneous.
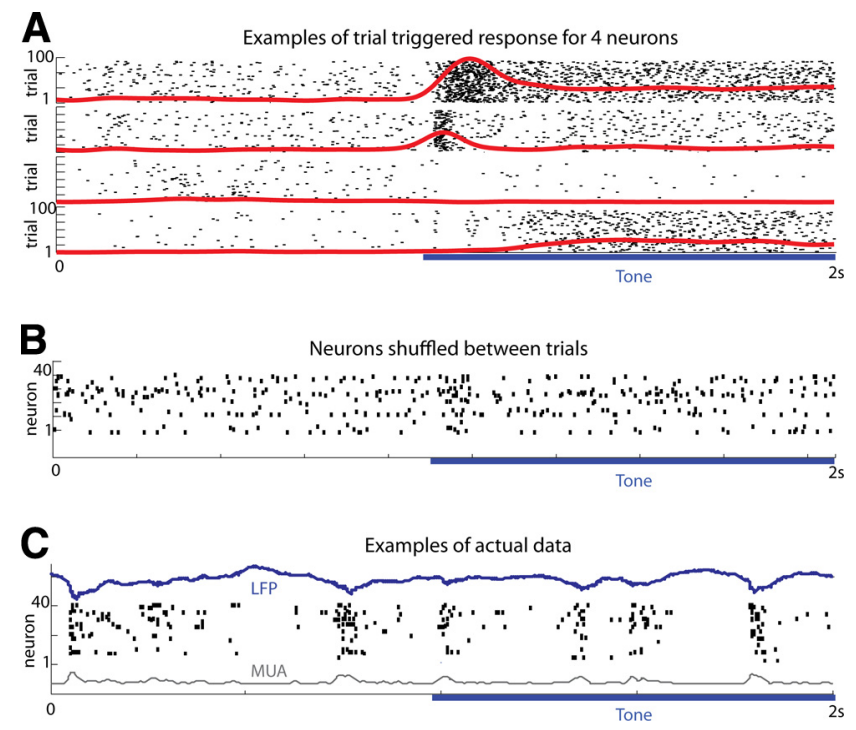

Figure 1. Responses of individual cells and populations to sustained tone stimuli. $\boldsymbol{A}$, Raster plots showing responses of four representative neurons to repeated presentations of a $1 \mathrm{~s}$ long $15 \mathrm{kHz}$ tone (100 trials shown for each neuron). Red lines represent smoothed PSTHs, and the blue line underneath indicates the duration of a tone stimulus. $\boldsymbol{B}, \mathrm{A}$ simulation of the structure of population activity expected if neurons were no more coordinated than predicted from the PSTHs. This plot was obtained by shuffling spikes of each cell between multiple stimulus presentations. C, Actual population activity on a single trial. The raster shows spikes of simultaneously recorded neurons and the blue trace shows local field potential. At bottom is the multiunit firing rate (MUA) computed as the smoothed summed activity of all neurons. Note that neurons tend to fire in transient bursts of 50-100 ms duration, with burst times including but not limited to tone onset.

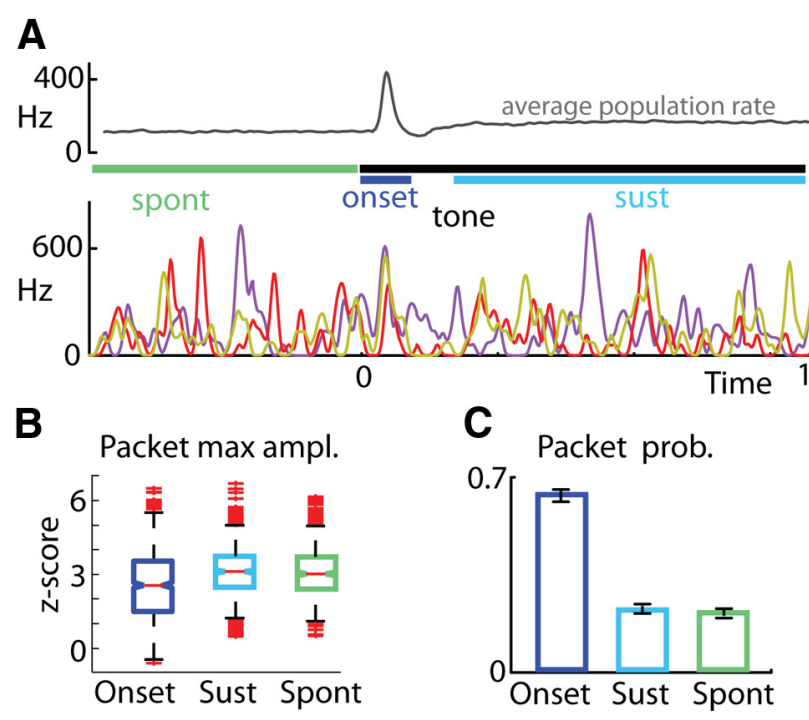

Figure 2. Population bursts have similar amplitude for onset, sustained, and spontaneous periods, but occur more reliably at stimulus onset. $\boldsymbol{A}$, Top, Trial-averaged population rate triggered at tone onset. Bottom, Examples of population rate on three single trials. Note that activity packets occur reliably at tone onset, but sporadically during spontaneous and sustained periods. $\boldsymbol{B}$, Box-andwhisker plots summarizing distributions of onset-evoked packets, and the distribution of amplitudes of the largest packets in the sustained and spontaneous periods. The central mark is the median, and the edges of the box are the 25th and 75th percentiles. C, Probability of occurrence of large activity packets (mean +2 SD) during spontaneous, onset, and sustained periods.

Fraction of time-locked spikes. To quantify the fraction of spikes time-locked to MUA, we first computed the area of the spiketriggered MUA above chance level. If the MUA were itself unstructured, this area would be proportional to the number of time-locked 
spikes from that neuron. To account for autocorrelation of MUA, the fraction $F$ of time-locked spikes was computed as $A_{\mathrm{xc}} / N_{1}{ }^{*} N_{\mathrm{MUA}} /$ $A_{\mathrm{MUA}}$, where $A_{\mathrm{xc}}$ is the area of the peak of the cross-correlogram (CCG), $A_{\mathrm{MUA}}$ is the area of peak of the MUA autocorrelogram, $N_{1}$ is number of spikes of the analyzed single neuron, and $N_{\mathrm{MUA}}$ is number of spikes in MUA. $F$ lies in the range 0 to 1 , with 0 indicating complete independence of the individual neuron from MUA and 1 indicating $100 \%$ time locking, i.e., that the neuron's instantaneous firing rate was proportional to instantaneous MUA rate.

The $\mu_{c c}$ measure. To quantify the fine temporal relationship between a neuron and population activity, we assessed the asymmetry of the spiketriggered MUA using a previously described measure, $\mu_{\mathrm{cc}}$, defined as the mean spike time of spike-triggered MUA within a $100 \mathrm{~ms}$ time window, which is equivalent to calculating the center of mass of a CCG (Luczak et al., 2009). Before calculating the center of mass, CCGs were normalized between 0 and 1 to discard effect of baseline activity. Analyses were repeated computing $\mu_{\mathrm{cc}}$ using multiunit activity taken only from each neuron's local recording shank; for all datasets, this resulted in changes in $\mu_{\mathrm{cc}}$ of $<5 \%$.

Information theoretic analysis. To estimate the amount of information transmitted by the population at each moment, we divided the sustained epoch of each tone presentation into 50-ms-long overlapping time periods. The periods were divided into 10 groups based on MUA rate, and the mean information content was estimated for each group by predicting the stimulus identity from firing rate vectors using linear discriminant analysis and evaluating predictability from the cross-validated confusion matrix (Narayanan et al., 2005). Use of other prediction methods such as multiclass support vector machines (one vs all mode, with Gaussian or polynomial kernels; www.codeforge.com/article/207841) (Cortes and Vapnik, 1995) and random forests (Breiman, 2001) (for more details, see Oh et al., 2003) gave similar results (data not shown).

Comparing sequential organization between tone frequencies. To determine whether sequential organization within sustained-period packets differed between tone frequencies, we used a method based on the $\mu_{\mathrm{cc}}$ measure. To allow comparison not just between frequencies, but also between multiple responses to the same frequency, we divided tone presentations into two groups, and for each group divided sustained-period responses into $50 \mathrm{~ms}$ bins. To ensure that sequential structure was assessed only from clear activity packets, we kept for each group only the $20 \%$ of periods with highest firing rate. Similarity between sequential structure was measured by the correlation across cells of $\mu_{c c}$ between Group 1 responses to the first tone frequency and Group 2 responses to the second. Sequence similarity was plotted as a function of distance between frequencies, based on tonal order.

A similar analysis was used to compute how sequential stereotypy varied as a function of firing rate (see Fig. 10B): for each firing rate group we computed a sequential similarity measure by correlating the average $\mu_{\mathrm{cc}}$ from all periods in the group with the $\mu_{\mathrm{cc}}$ in the spontaneous period. To verify that these results did not just reflect that sequential order can be more reliably evaluated with more spikes, we equalized the number of spikes in all groups by randomly removing spikes and then recalculated sequential order.

\section{Results}

\section{Structure of population responses to extended tones}

We used silicon microelectrodes to record from neuronal populations of deep layers of primary auditory cortex, in four headfixed passively listening rats, while presenting repeated 1-s-long tone stimuli. Figure $1 A$ shows raster representations of four representative neurons' responses to multiple repetitions of a $15 \mathrm{kHz}$ tone, together with peristimulus time histograms (PSTHs) showing each neuron's mean firing rate as a function of time into the stimulus. Measured PSTHs were consistent with previous reports, typically showing transient frequency-tuned responses at onset, and rarer increases in firing rate during the sustained period (Vaadia et al., 1982; Wang et al., 2005; Moshitch et al., 2006; Bartho et al., 2009). Of the 120 neurons in our database, $63 \%$
A
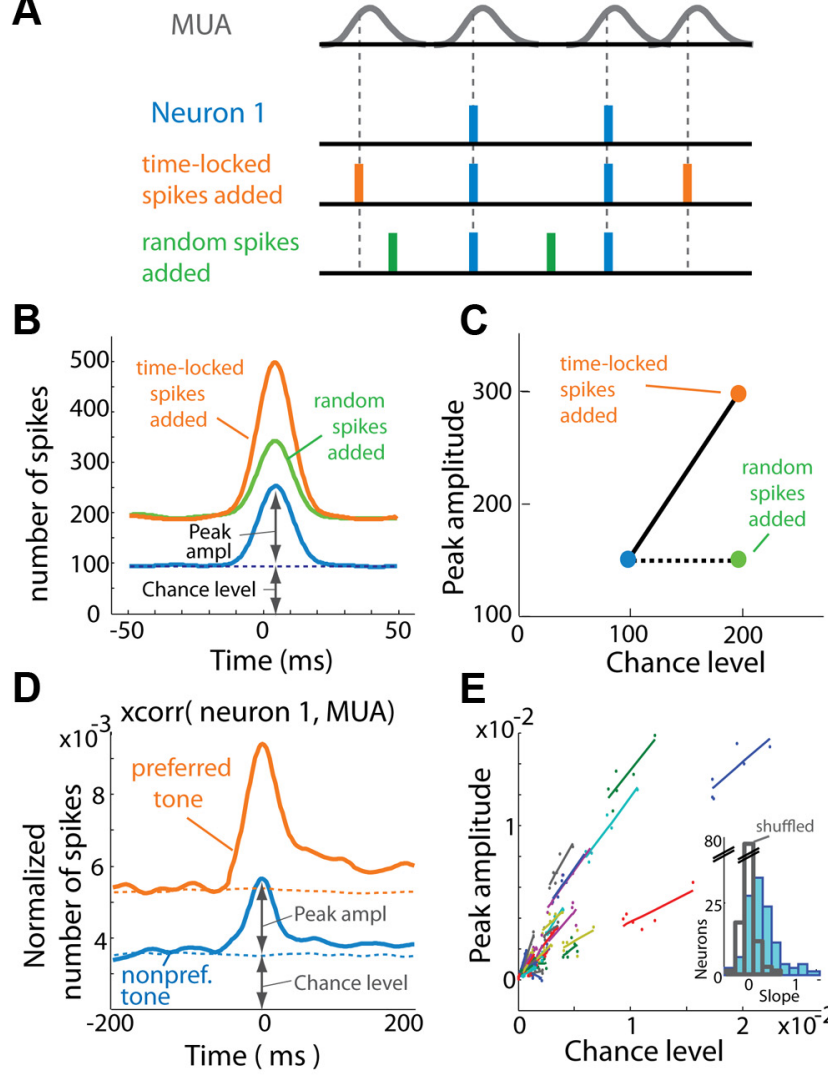

E
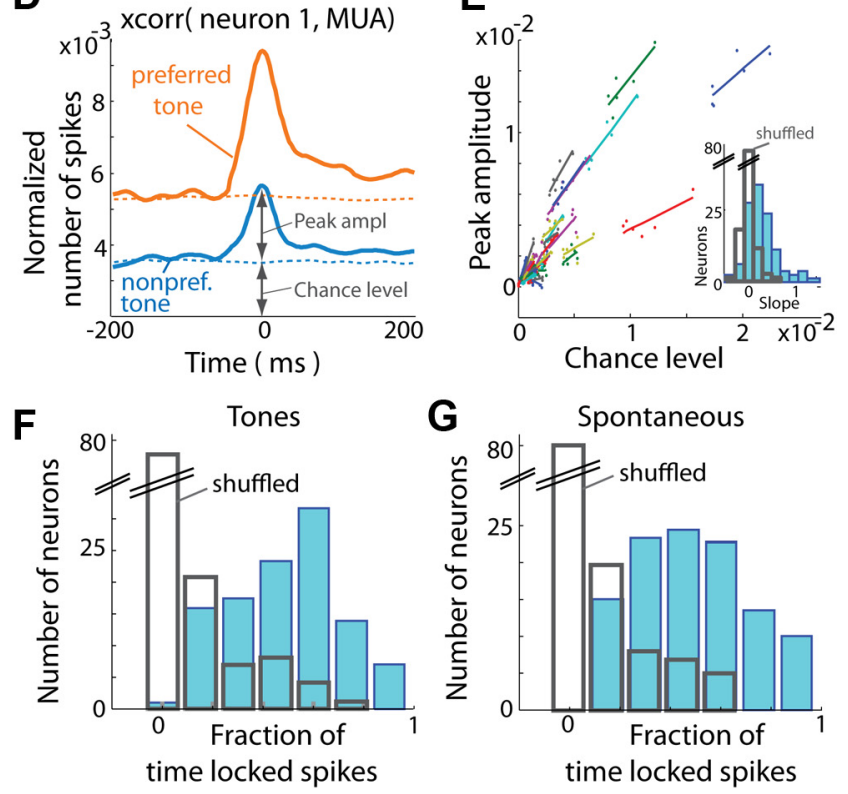

Figure 3. Sustained preferred tones cause increased firing locked to spontaneous activity. $\boldsymbol{A}$, Diagram illustrating how sustained tone presentation could affect an individual neuron's firing in the context of population activity. Gray curve (top) indicates population MUA rate. Blue indicates a hypothetical neuron's activity in the absence of sensory stimuli. Red indicates additional spikes due to tone presentation, locked to spontaneous MUA fluctuations. Green indicates additional spikes occurring at random times unrelated to spontaneous activity. $\boldsymbol{B}$, CCGs between a single neuron and MUA, generated from data simulated according to the above scenarios. Adding random spikes (green) increases the CCG baseline level, but does not affect the area of the CCG peak, defined as the sum of activity above baseline level within the -50 to +50 ms time window. Adding the same number of spikes time locked to MUA (red) increases both the baseline level and the peak. $\boldsymbol{C}$, Plotting peak amplitude versus baseline level results in positive slope if time-locked spikes are added, and zero slope for randomly added spikes. $\boldsymbol{D}$, Example CCG between a recorded neuron and MUA, for its preferred tone and a nonpreferred tone. Dashed lines represent baseline levels calculated from trial-shuffled data. Note that the amplitude of the peakscales up as the baseline level increases, indicating addition of spikes time locked to MUA. $\boldsymbol{E}$, Relationship between baseline level and peak amplitude for all neurons from one experiment. Each dot represents the response of one neuron to one tone, with colors indicating different neurons, and lines fitted for each neuron by linear regression. Inset, Histogram of regression slopes across all experiments (blue bars) and from trial-shuffled data (gray bars). $\boldsymbol{F}$, Fraction of time-locked spikes across all experiments during sustained periods of tone presentations. Gray line shows trial-shuffled data. $\boldsymbol{G}$, The same plot as in $\boldsymbol{F}$ but for spontaneous periods. 

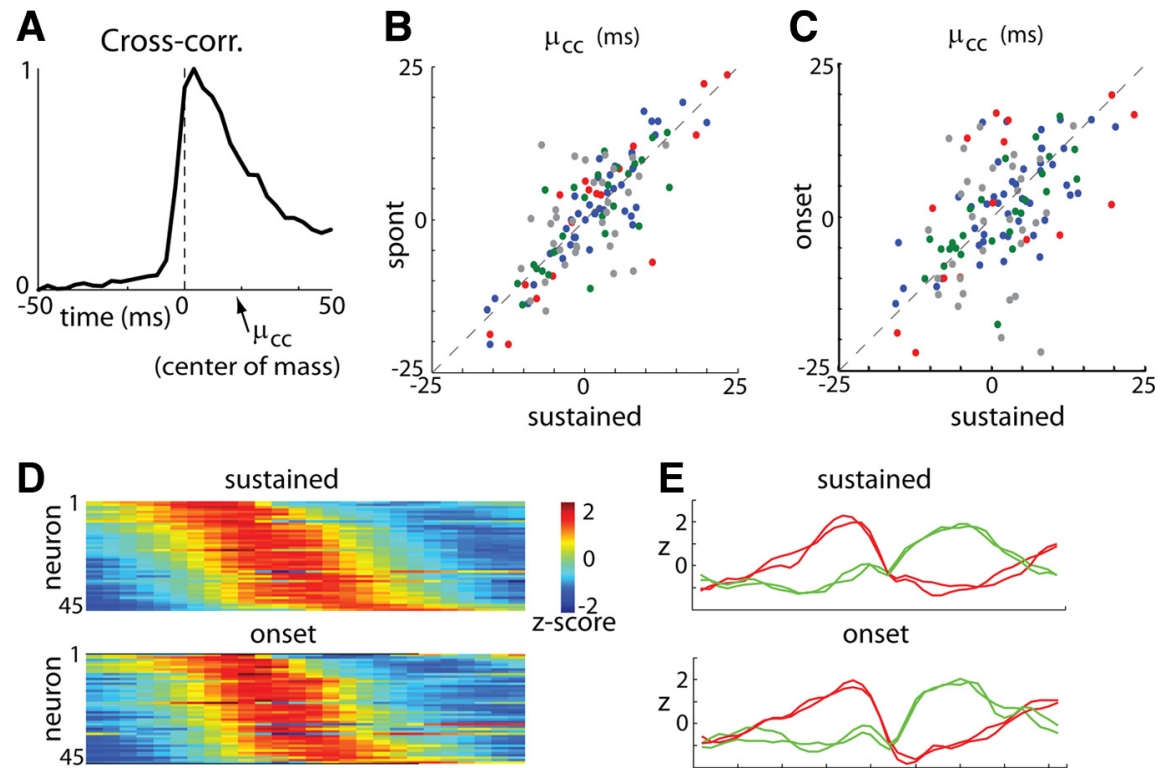

onset
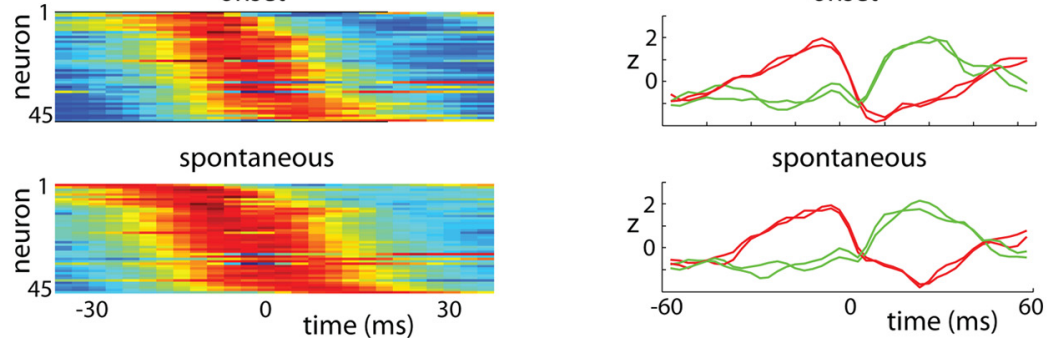

Figure 4. Temporal relationships between neurons are conserved across conditions. $\boldsymbol{A}$, The timing of each neuron relative to the population was summarized by a $\mu_{c c}$ measure, computed as the center of mass of the scaled spike triggered MUA (CCG). The CCG shown has a rightward skew, indicating that this neuron fired at the beginning of periods of elevated population activity. $\boldsymbol{B}$, The $\mu_{c c}$ measure for each neuron calculated during sustained tone responses and during spontaneous activity. Neurons from different animals are shown with different colors. The distribution of points along the equality line shows that each neuron's temporal relationship to the population is preserved across conditions. C, Comparison of $\mu_{\mathrm{cc}}$ for onset responses and sustained periods. D, Pseudocolor representation of CCGs for all neurons of a representative experiment, in three conditions. Each horizontal line of the pseudocolor matrix corresponds to the CCG of one neuron, vertically arranged in the same order for each plot, according to the value of $\mu_{\mathrm{cc}}$ in the sustained period. For visualization, CCGs are normalized to mean 0 and unit variance. $\boldsymbol{E}$, Line plot of normalized CCGs for the neurons with the smallest (red) and largest (green) values of $\mu_{c c}$. To illustrate stability of CCGs within an experimental condition, two curves are superimposed for each cell, showing CCGs calculated from 50\% of trials in each condition.

showed a significant dependence of sustained firing rate on tone frequency $(p<0.05$, Kruskal-Wallis test). This dependence did not simply consist of excitatory responses, with $36 \%$ of cells showing a significant increase in rate to at least one tone, but $54 \%$ showing a significant decrease $(p<0.05$; Kolmogorov-Smirnov test).

What patterns of population activity occur during these single-neuron responses? Figure $1 A$ appears to suggest that after a transient onset response, each neuron settles down to fire steadily at a constant rate until the end of the tone. If this were the case, population activity would have the structure shown in Figure $1 B$, which was generated by shuffling individual spikes between stimulus presentations (trial shuffling) to produce a conditionally independent Poisson process with the same rate profile as the original data. Figure $1 C$ shows a raster representation of an actual population response to a tone stimulus. Rather than showing constant rate, spontaneous fluctuations in global activity are apparent, with transient packets of high population rate interleaved with periods of near-total inactivity. Activity packets were accompanied by deflections in LFP, suggesting an increase in coordinated synaptic activity during those times. The timing of the active and inactive periods varied considerably from trial to trial. However, the global activity pattern was not strongly affected by tone presentation. Indeed, if the periods of tone presentation were not marked on Figure $1 C$, it would be impossible to visually combined onset responses of a large number of cells, followed by a smaller sustained rate elevation. Figure $2 A$ (bottom) plots the single-trial population rate for three individual presentations of the same tone. In this view, the packets occurring at onset do not appear larger than those occurring spontaneously during sustained tones or silence. To quantify this, we calculated, for each tone presentation, the height of the population rate peak after stimulus onset $(0-100 \mathrm{~ms})$, the height of the highest population rate peak in the preceding period of silence $(-800$ to $0 \mathrm{~ms}$, to avoid offset responses from the previous tone), and the highest population rate peak in the sustained response period (200 ms to $1 \mathrm{~s}$ after tone onset). Population rates at onset were not larger than the highest peaks during $800 \mathrm{~ms}$ immediately before or after onset response at each trial, indicating that the population was typically more active at some moment during the sustained period or silence preceding each tone than at onset (Fig. $2 B ; \mathrm{Ampl}_{\text {onset }}=2.5, \mathrm{Ampl}_{\text {sust }}=3.1, \mathrm{Ampl}_{\text {spont }}=3.0$, rates are expressed as $z$ scores to combine data from different experiments; $p_{\text {onset }- \text { sust }}<0.0001, p_{\text {onset-spont }}<0.0001$, $p_{\text {sust-spont }}=0.2$, two-sample Kolmogorov-Smirnov test). However, the probability of seeing an activity packet (measured as instantaneous MUA rate larger than mean +2 SD) was significantly higher at onset compared to any time point during spontaneous or sustained periods (Fig. $2 C$; Prob $_{\text {onset }}=$ 0.63, Prob $_{\text {sust }}=0.22$, Prob $_{\text {spont }}=0.21 ; p_{\text {onset }- \text { sust }}<0.0001$, $p_{\text {onset }- \text { spont }}<0.0001, \quad p_{\text {sust }- \text { spont }}=0.92$, two-sample 

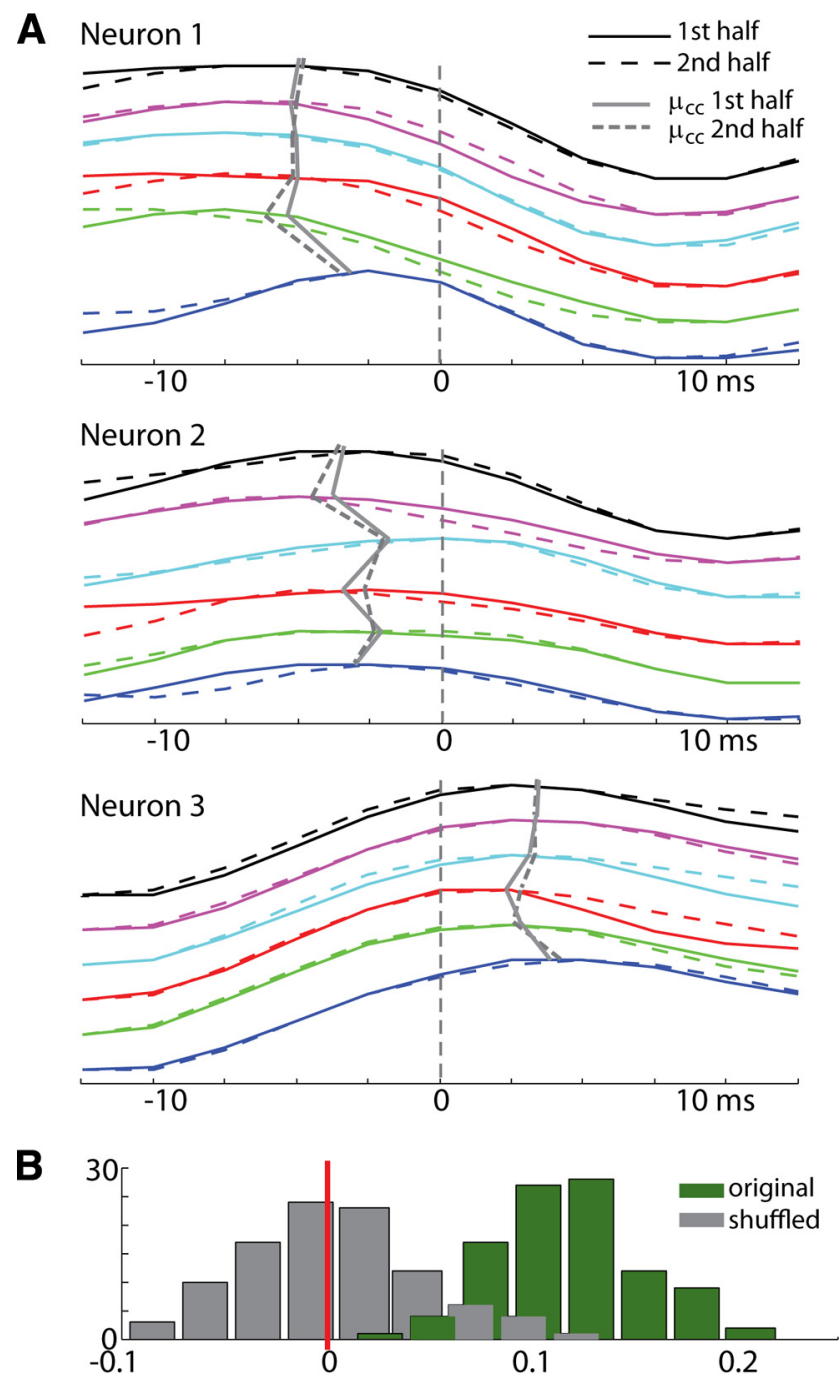

similarity of xcorrs to the same tone - other tones $(\Delta$ corr coef)
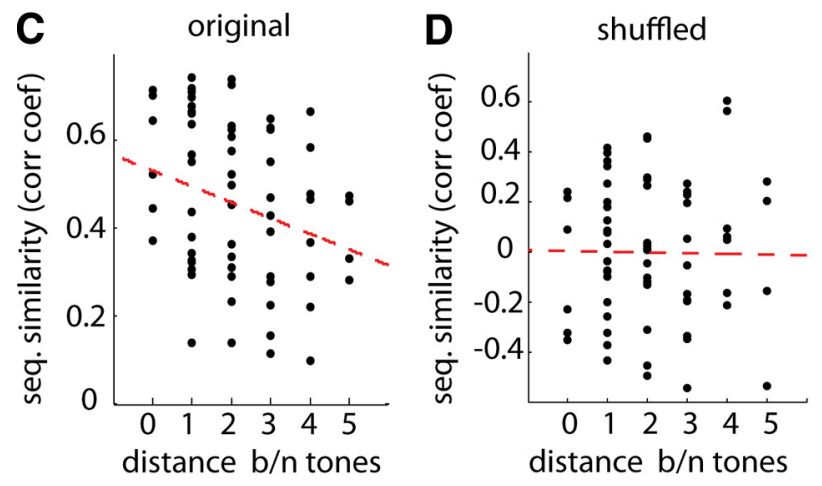

Figure 5. Effect of tone frequency on temporal fine structure during sustained period. $A$, Examples of how tone frequency affects the cross-correlogram between a single neuron's spikes and summed population activity. The three panels show data for three representative neurons. The six colors in each panel represent CCGs computed in the sustained periods of six different tone frequencies, with solid and dashed curves showing CCGs computed independently from two halves of the data set to illustrate reliability. The solid and dashed gray lines show the values of $\mu_{\mathrm{cc}}$ as a function of frequency for the two halves. Note that timing differences between neurons are larger than differences between frequencies for a single neuron, but the latter also consistently occur. $\boldsymbol{B}$, To quantify the modulation of $\mathrm{CCG}$ shape by tone frequency, we compared the similarity of $C(G$ shapes for a single frequency between two halves of the data set, relative to similarity between different frequencies. The green histogram shows this quantity averaged over cells, for 100 different random splits of the data set, indicating that CCG shapes differ
Kolmogorov-Smirnov test). To verify that these results did not simply result from occasional random spiking coincidences during the sustained and spontaneous periods (which are longer than the onset periods), we performed the same analyses on trial-shuffled data (as in Fig. 1B). After shuffling, the peak population rate of spontaneous and sustained activity was now smaller than onset response $\left(\mathrm{Ampl}=2.2 ; \mathrm{Ampl}_{\text {sust shuf }}=1.6 ; \mathrm{Ampl}_{\text {spont shuf }}=1.5\right.$; rates expressed as $z$ scores of original MUA), and sustained and spontaneous but not onset event probability was much smaller than in the actual data $\left(\right.$ Prob $_{\text {onset shuf }}=0.65$ Prob $_{\text {sust shuf }}=0.04$; Prob $\left._{\text {spont shuf }}=0.04\right)$, confirming that the detected activity packets reflected true synchronous firing events. We therefore conclude that the onset responses seen in PSTHs and single-neuron rasters reflect a stimulus-locked increase in the probability of activity packets, rather than an increase in their size.

\section{Tone-evoked spikes are locked to spontaneous activity packets}

The above data suggest that population activity during sustained tone presentations is composed of spontaneous packets of similar size to tone onset responses. However, in our data as in previous reports, analysis of single-neuron PSTHs indicates that sustained responses consist of an increase in trial-averaged mean rate throughout the duration of a tone presentation (e.g., Fig. 1A). How is the timing of additional action potentials evoked by a sustained tone related to ongoing spontaneous activity packets? We considered two possibilities, both consistent with the observed PSTHs of individual neurons (Fig. $3 A$ ). First, the extra spikes could occur specifically during packets of high network activity ("time-locked" spikes); second, they could occur evenly throughout the tone presentation, without regard to global network activity ("random spikes").

To distinguish between these possibilities, we used an analysis based on the spike-triggered MUA for each cell, computed as the CCG of an individual neuron's spike train, with the summed multiunit activity of all other neurons. Figure $3 B$ illustrates the effect of tone presentation on spike-triggered MUA, using data simulated according to the two models considered. For timelocked spikes, tone presentation would increase both the baseline level and peak amplitude (i.e., baseline-to-peak height) of the spike-triggered MUA; for random spikes, tone presentation would increase the baseline level only (Fig. 3C). Figure 3D shows actual data for a single recorded neuron, for which spiketriggered MUA during presentation of a preferred tone (brown) showed a robust increase in both baseline and baseline-to-peak height, compared to presentation of a nonpreferred tone (blue; to avoid a possible confound caused by changes in MUA rate for different tones, MUA traces were normalized to unit area for this analysis; note also the asymmetry of both CCGs, which will be discussed further below). Figure $3 E$ summarizes the data for all neurons recorded in this experiment, showing a robust relationship of baseline level to peak amplitude, a result that was borne out across all experiments (Fig. 3E, inset; average slope of baseline

$\leftarrow$

significantly between frequencies. The gray histogram shows a control analysis performing the same procedure after tone identity was shuffled. C, Sequential structure of sustained population activity depends on tone frequency. Sequential similarity was measured as the correlation coefficient of $\mu_{c c}$ across the population (Fig. $4 B, C$ for all pairs of tone frequencies. Note that greatest similarity is seen between responses to different presentations of the same frequency (distance 0 ), whereas a smaller but nonzero similarity is seen for widely separated tone frequencies (distance 5). D, The same analysis computed after trial shuffling to remove interactions between cells (Fig. 1B). After shuffling, sequence similarity is zero for all tone distances. 


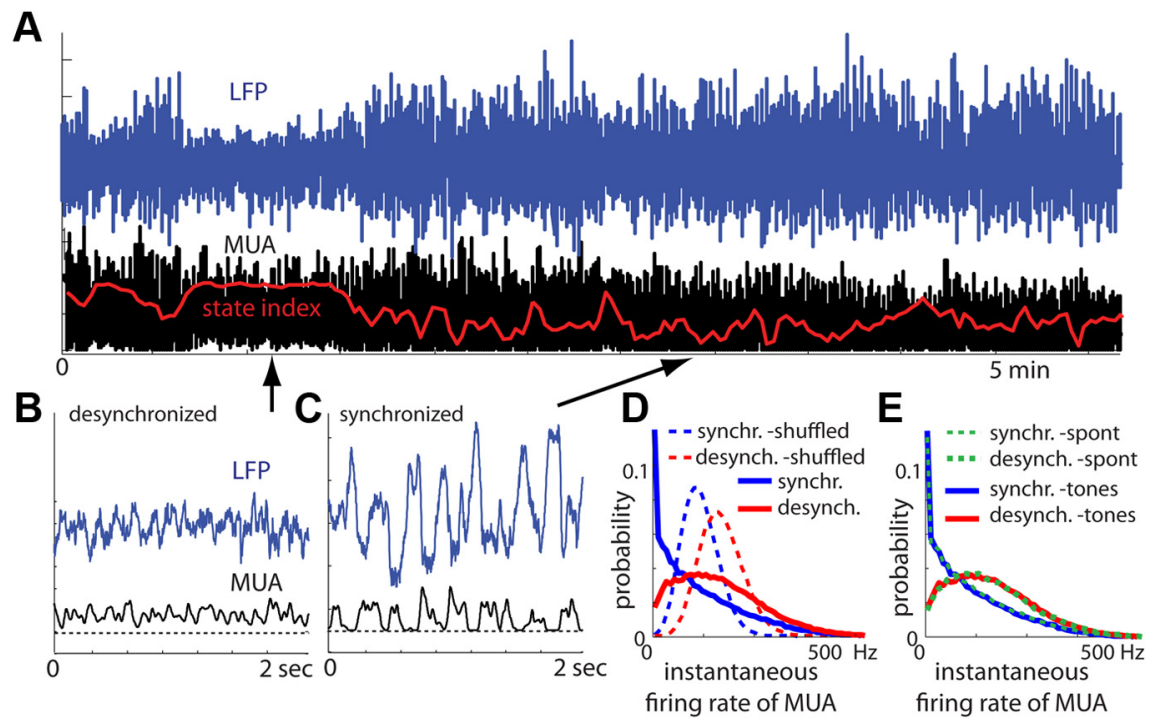

$\mathbf{F}$

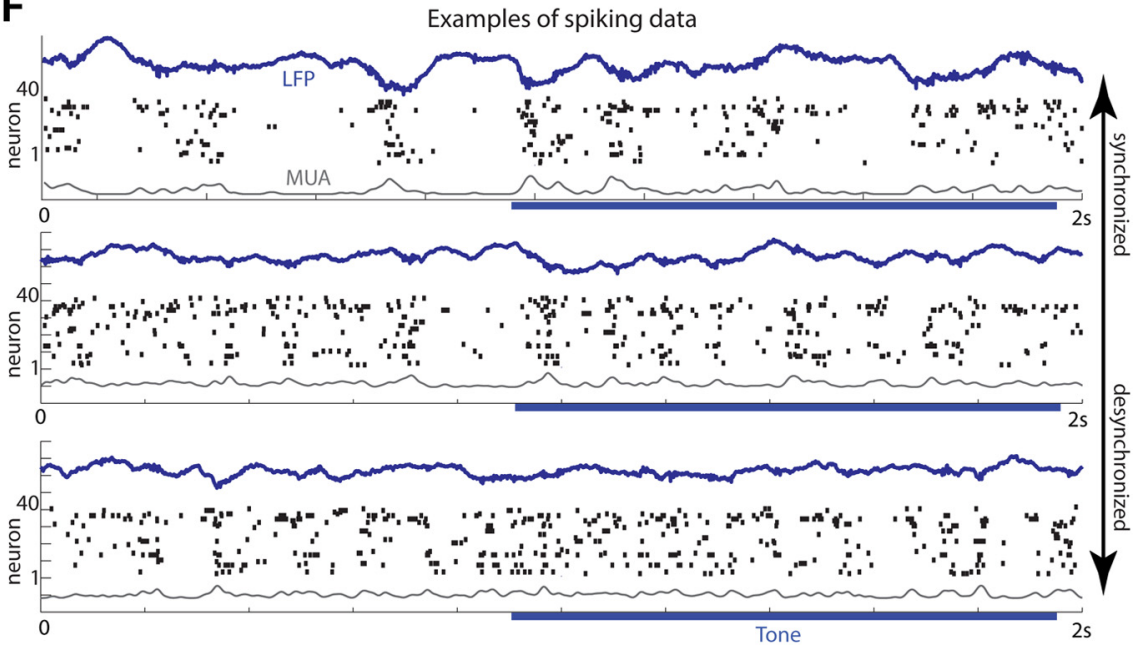

Figure 6. Effect of cortical state on sustained-period activity. A, Raw LFP trace (blue) and MUA firing rate (black) during several minutes of acoustic silence. Red curve shows the "state index," defined as the percentage of time bins for which MUA rate was nonzero. $\boldsymbol{B}, \boldsymbol{C}$, Expanded views from the periods marked by arrows. The dashed horizontal line indicates a zero MUA rate. $\boldsymbol{D}$, Histogram of instantaneous MUA rates during sustained tone responses, for desynchronized and synchronized trials. Dotted lines indicate the same analysis for trial-shuffled data. $\boldsymbol{E}$, Comparison of MUA rate histograms for the sustained ( $200 \mathrm{~ms}$ to $1 \mathrm{~s})$ period of tone presentation (solid lines) and for spontaneous activity (dashed). $\boldsymbol{F}$, Examples of single-trial raster plots sorted by cortical state (conventions as in Fig. 10). Activity during desynchronized states shows weaker global fluctuations but still exhibits complex fine structure.

level vs peak amplitude $0.3 \pm 0.34 \mathrm{SD}$, significantly larger than trial-shuffled data of $0.03 \pm 0.03 \mathrm{SD} ; p<0.001$, two-sample Kolmogorov-Smirnov test). This analysis suggests that the additional spikes a neuron fires in response to its preferred tone are primarily aligned with spontaneous activity packets. To estimate the fraction of spikes locked to MUA, we used a measure based on the area of the CCG above baseline level, normalized by the MUA autocorrelogram (see Materials and Methods). Figures 3, $F$ and $G$, shows values of this fraction from sustained and spontaneous periods, which are both significantly larger than for trial-shuffled data $(p<0.001)$, confirming that most tone-evoked spikes occur within activity packets.

Sustained-period activity packets have a broadly conserved sequential structure with further variations between tone frequencies

Activity packets evoked by tone onsets have a temporal structure that is largely conserved between stimuli and shows a further but smaller variation depending on tone frequency, such that the difference in timing of a single cell between stimuli is smaller than the difference between multiple cells to a single stimulus (Luczak et al., 2009). We next asked whether a similar organization was found for sustained-period packets. This requires answering two questions: first, do sustained-period packets have a temporal structure that is broadly conserved across stimuli? And second, are there further variations on this structure depending on sustained tone frequency?

The temporal structure of sustainedperiod activity packets cannot be analyzed by examining trial-averaged activity, due to the unpredictable timing with which sustained-period packets occur. We therefore adopted an analysis method described previously (Luczak et al., 2007, 2009), in which the temporal relationship of each neuron to the rest of the population is summarized by a measure $\mu_{\mathrm{cc}}$, the center of mass of its spike-triggered MUA, i.e., its CCG with the summed population activity of all other neurons (Fig. 4A). Visual examination of spike-triggered MUA revealed a common occurrence of asymmetrical shapes, indicating that individual neurons typically fired in a stereotyped temporal position compared to the rest of the population (the neuron in Fig. $4 A$ for example, typically fired at the start of population activity packets). Asymmetrical CCGs occurred both for neuronal pairs recorded on the same shank and on different shanks, consistent with previous work indicating that these CCGs reflect temporal heterogeneity within local populations rather than the spreading of traveling waves across the cortical surface (Luczak et al., 2007). Values of $\mu_{\mathrm{cc}}$ were strongly correlated between spontaneous and evoked activity (Fig. $4 B ; r=0.75 \pm 0.21$ SD), and between onset (0-100 ms) and sustained (200-1000 $\mathrm{ms}$ ) periods of tone presentations (Fig. $4 C ; r=0.53 \pm 0.23 \mathrm{SD}$ ). Visualization of spike-triggered MUA indicates a continuum of timing profiles in the population, preserved between and within conditions, spanning a time course of $\sim 50 \mathrm{~ms}$ (Figs. $4 D, E$ ). These results indicate that activity packets occurring during sustained tones exhibit a broadly conserved sequential structure of time scale $50-100 \mathrm{~ms}$, which is similar to the structure of activity packets occurring at tone onset and during acoustic silence.

We next asked whether different tone stimuli caused variations around this common sequential structure. Figure $5 \mathrm{~A}$ shows plots of spike-triggered MUA computed during the sustained period for six tone frequencies, for three example neurons. As expected from the analyses of Figure 4, differences in CCG shape between neurons are typically larger than differences within a single neuron's response to the different tones. Nevertheless, these plots suggest that further differences between tone frequencies do exist. To test this idea statistically, we asked whether the 
differences between CCG shapes evoked by different tones were significantly greater than the random variations in CCG shapes for a single tone estimated from different halves of the data set (Fig. $5 A$, solid and dotted lines). The green histogram in Figure $5 B$ shows the distribution differences between within-tone and between-tone similarities (assessed by the correlation coefficient of CCG shapes) for 100 random halvings of the data set, indicating a consistent difference from zero $(p<0.001 ; t$ test); a control analysis performed after shuffling tone identity showed no effect (gray histogram; $p=$ 0.62 ). To complement this analysis of single-cell timing relative to MUA, we performed a second analysis that assessed the similarity of the sequential structure of the entire recorded population by correlating $\mu_{\mathrm{cc}}$ values of all cells between responses to the one tone frequency on the first half of the data set, and to another on the second half. Figure $5 C$ shows the mean sequence similarity as a function of distance between frequencies, based on tonal order. Sequences become less correlated with increasing frequency difference between tones (Fig. $5 C ; r=-0.22 \pm$ 0.08 SD, $p<0.007$ for pooled data, $p<$ 0.02 for three of four rats individually, $p=$ 0.08 for the fourth rat). This effect was not observed for shuffled data $(r=-0.02, p=$ 1; Fig. 5D). Thus, as with onset-evoked packets, sustained-period packets have a broadly conserved 50 $100 \mathrm{~ms}$ sequential structure, but this structure exhibits further variations depending on tone frequency.

\section{Cortical state determines the distribution of activity packets}

In quiescent waking animals, such as studied here, cortical spontaneous activity exhibits a range of states corresponding to different levels of ongoing fluctuation (Harris and Thiele, 2011). To determine how these states affected population spiking patterns, we first computed for each trial a "state index," defined to be the percentage of time in which the smoothed multiunit firing rate was not zero. Figure $6 \mathrm{~A}$ shows how this measure varied over several minutes of spontaneous activity (no tones presented), together with raw LFP and MUA traces. Cortical state is not discrete, but a continuum; however, to simplify the analyses below, trials were divided into a "synchronized" group and a "desynchronized" group, corresponding to the two extreme thirds of the distribution, and an intermediate group that was not used for further analysis. Short data segments from each group are expanded in Figure 6, $B$ and $C$.

Figure $6 D$ shows histograms of the distribution of multiunit firing rate for the synchronized and desynchronized trials during the sustained period of tone presentations (from $200 \mathrm{~ms}$ after onset until offset). For synchronized trials, the histogram (blue) shows a clear mode at 0 , indicating the presence of prolonged down phases, and a "tail" corresponding to up phases of global network activity. This is in marked contrast to a similar histogram obtained from the same data shuffled across trials (blue dashed), confirming that these fluctuations were much stronger
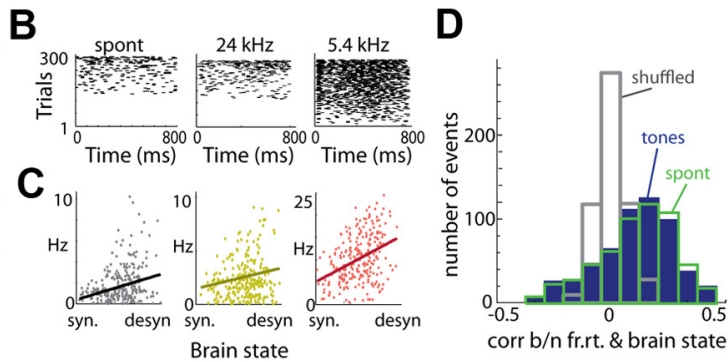

Brain state

$\mathbf{F}$

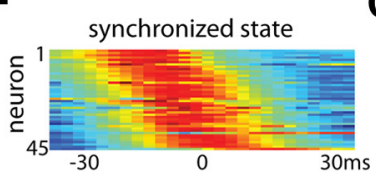

G
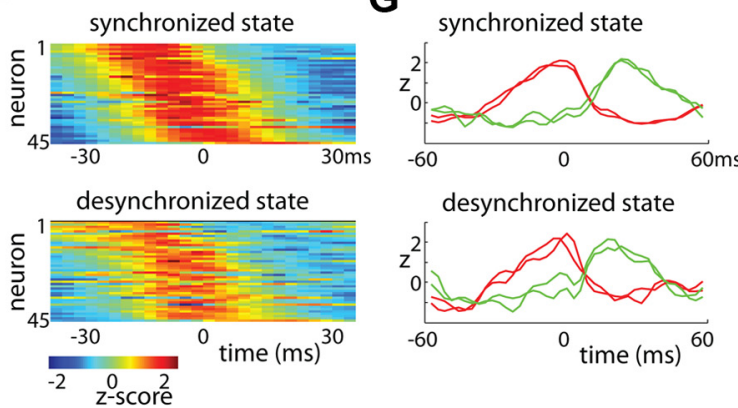

desynchronized state

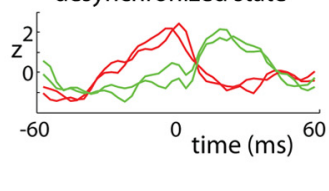

Figure 7. Firing rates are modulated by both state and stimuli, but temporal constraints are conserved across states. $\boldsymbol{A}$, Raster showing responses of a neuron to 100 repetitions of each tone, arranged by presentation order. Colored lines denote all tones. Correlations for sustained tone responses (blue) and spontaneous epochs (green) are similar to each other, but differ

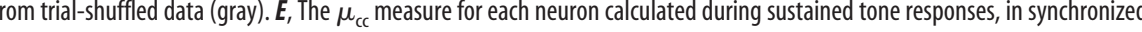
synchronized and desynchronized periods, sorted in the same vertical order. G, Corresponding examples of CCGs with the smallest (red) and largest (green) $\mu_{c c}$ measures, as in Figure $4 E$.

than expected by chance. For desynchronized trials, the distribution of MUA was less skewed, with no mode at zero, but still different from the shuffled data, confirming the existence of smaller but still significant fluctuations in the desynchronized state ( $p<0.001$, two-sample Kolmogorov-Smirnov test). Figure $6 E$ shows a superimposition of the MUA rate histograms computed from sustained tone responses (solid lines), and from spontaneous activity occurring in the epochs immediately before sound presentation (dashed lines), for desynchronized and synchronized trials. In both cases, very close overlap is seen between the activity during the sustained and silent periods. This is also illustrated in single-trial examples in Figure $6 F$, suggesting that cortical state, rather than tone presentation, primarily determines the structure of the global fluctuations.

We next asked how cortical state shaped individual neurons' responses to tone stimuli. Figure $7 A$ shows a conventional raster representation of a single neuron's response to 100 repetitions of five frequencies of tone. While these plots may give the impression that the neuron fires steadily during the sustained period of tone response, this is again an incomplete picture. Figure $7 B$ shows another view of the same data, in which the responses of this cell to individual stimulus repetitions have been ordered vertically by mean firing rate during the sustained period, revealing a high level of variability from trial to trial. Correlation analysis showed that much of this variability reflected differences in cortical state between trials (Fig. $7 C ; p<0.01$; state index was calculated from the $1 \mathrm{~s}$ of data before and after tone presentation, to avoid confounding with firing rate during the tone). This effect was borne out in general (Fig. 7D), with correlations between 
firing rate and state index significantly more positive than trialshuffled data for both tone-evoked and spontaneous periods $(p<0.001$, two-sample Kolmogorov-Smirnov test), and the mean level of correlation slightly higher during silent than sustained tone periods ( $p=0.014$ ). This shows that the firing rate of neurons can be as much affected by brain state as by stimuli.

The fact that the desynchronized state does not exhibit large global fluctuations does not imply that cortical population activity in this state has no temporal fine structure (Renart et al., 2010; Harris and Thiele, 2011). To investigate this question, we again employed the $\mu_{\mathrm{cc}}$ measure. The $\mu_{\mathrm{cc}}$ measure was strongly correlated between trials in synchronized and desynchronized states (Fig. $7 E ; r=0.67 \pm 0.23 \mathrm{SD}$ ), and the temporal response profiles of individual neurons were preserved between and within conditions (Figs. $7 F, G$ ). Thus, even though the desynchronized state does not show strong fluctuations in mean activity or clear up and down phases, timing relationships between neurons are preserved in this state.

\section{Relation to local field potential}

The above analyses have indicated that a given neuron fires with largely stereotypical timing relative to the 50-to 100-ms-long activity packets that build sustained-period activity. Because summed population activity is strongly correlated with the (negative) local field potential (Fig. 6), we asked how a neuron's timing with respect to these packets was related to its phase of firing with respect to the LFP. Because of the $\sim 100 \mathrm{~ms}$ time scale of the activity packets, we first focused on a reciprocal frequency band of 8-12 Hz. Visual examination of example neurons supported a relation between spike timing with respect to MUA and LFP phase. The neuron shown in Figure $8 A-C$, for example, fired at the start of activity packets, as evidenced by the positive time offset of the spike-triggered MUA peak (Fig. 8A). This neuron fired on the falling phase of the LFP, as evidenced both by the spike-triggered average LFP (Fig. $8 \mathrm{~B}$ ) and phase measured with respect to LFP filtered in the $8-12 \mathrm{~Hz}$ band (Fig. $8 \mathrm{C}$ ). Figure $8 D-F$ shows the same plots for second neuron that fired later with respect to MUA, and on the rising phase of LFP. The timing and preferred LFP phase of both neurons was preserved between cortical states, although modulation depth was lower in the desynchronized state. The visual impression suggested by these neurons was confirmed at the population level by a relationship between the $\mu_{\mathrm{cc}}$ measure and mean firing phase with respect to $8-12 \mathrm{~Hz}$ LFP (Fig. 8G; $R_{\mathrm{syn}}=0.73 \pm 0.17 \mathrm{SD}, p_{\mathrm{syn}}<0.05 ; R_{\mathrm{des}}=$ $0.65 \pm 0.28 \mathrm{SD}, p_{\mathrm{des}}<0.05$; circular-linear correlation) (Berens, 2009), indicating that preferred firing phase in this frequency range primarily reflects timing with respect to the spontaneous activity packets.

We next asked which LFP frequency bands are most strongly related to spike timing. Figure $9 A$ shows the mean LFP power spectrum in both states. No peak was observed, confirming the lack of any clear narrowband oscillation in either state; nevertheless, a difference between states was seen, with low-frequency $(<20 \mathrm{~Hz})$ power reduced in the desynchronized state $(p=0.03$, paired $t$ test) together with a trend toward increased gamma frequency power $(50-80 \mathrm{~Hz} ; p=0.08$, paired $t$ test $)$. Examination of spike-field coherence showed a clear peak in the $5-20 \mathrm{~Hz}$ range that was most prominent in the synchronized state, but still present in the desynchronized state. This suggests that the locking of spikes to LFP is most prominent for frequency ranges corresponding to the $50-100 \mathrm{~ms}$ time scale of activity packets. To investigate whether the relationship of $\mu_{\mathrm{cc}}$ and LFP phase showed a similar dependence on frequency, we repeated the circular-
A
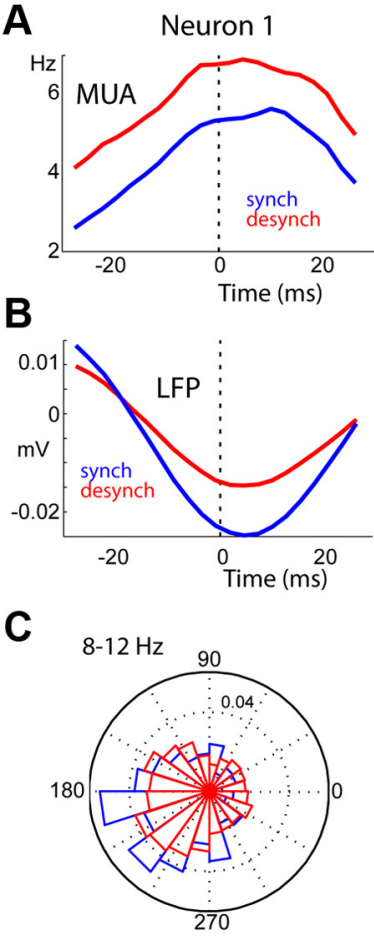

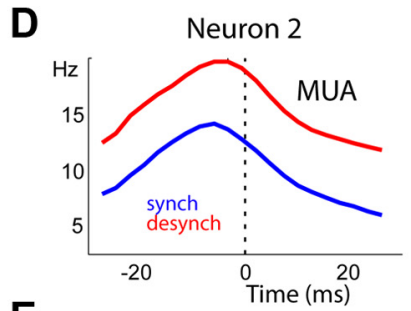

E

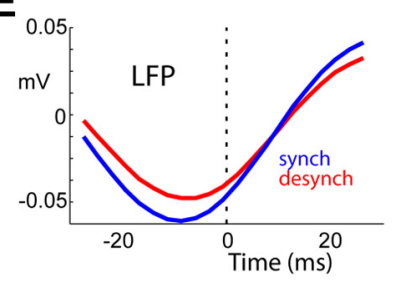

$\mathbf{F}$

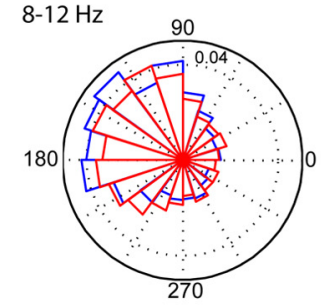

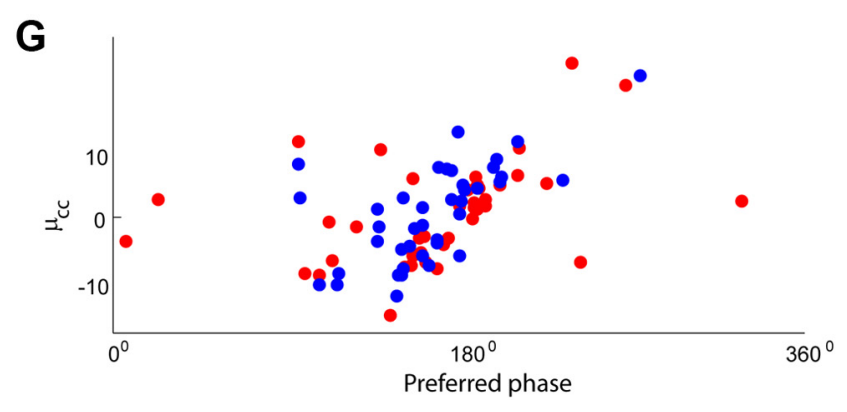

Figure 8. Phase relationship of spike timing to LFP mirrors timing relationship to population activity. $\boldsymbol{A}$, Spike-triggered MUA histogram for an example neuron, computed in the synchronized (blue) and desynchronized (red) states. $\boldsymbol{B}$, Spike-triggered LFP for the same neuron. Note that the (negative) peaks of these curves occur at a similar time to the peaks of the spiketriggered MUA in $\boldsymbol{A}$. $\boldsymbol{C}$, Histogram of spike phases for the same neuron with respect to LFP filtered in the 8-12 Hz frequency band in synchronized (blue) and desynchronized (red) states; note the similar phase preference across states, but deeper modulation in the synchronized state. $\boldsymbol{D}-\boldsymbol{F}$, Same plots as $\boldsymbol{A}-\boldsymbol{C}$ for a different neuron recorded in the same experiment. Note the similarity of each neuron's timing relative to LFP and MUA, but consistent differences between neurons. $\boldsymbol{G}$, Relation between $\mu_{\mathrm{cc}}$ and mean LFP phase at 8-12 Hz. Each dot represents a neuron during synchronized (blue dots) and desynchronized (red dots) periods.

linear correlation of Figure $8 G$ for a range of frequency bands (Fig. 9C). Similarly to spike-field coherence, a strong peak was seen close to $10 \mathrm{~Hz}$, which was larger in the synchronized state, but still present in the desynchronized state. Preservation of mean firing phases between synchronized and desynchronized states was also strong in low frequencies, but weak in higherfrequency bands (Figs. 9D,E). We thus conclude that firing phase with respect to LFP does indeed reflect timing with respect to activity packets, but only for frequencies below $\sim 20 \mathrm{~Hz}$, as expected from the superimposition of activity packets of length $50-100 \mathrm{~ms}$.

\section{Information content during sustained period}

The above analyses have shown that firing rates during the sustained period fluctuate from moment to moment, with the na- 
A

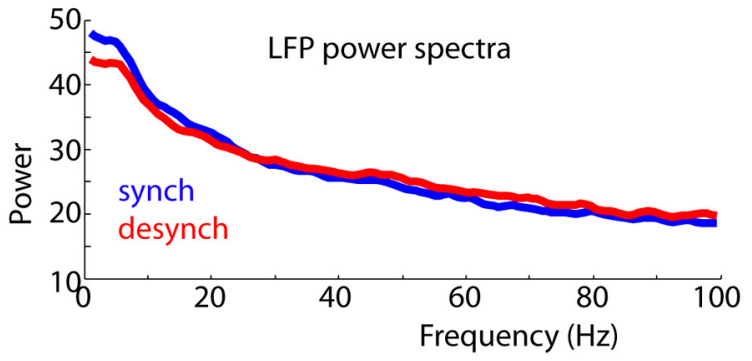

B

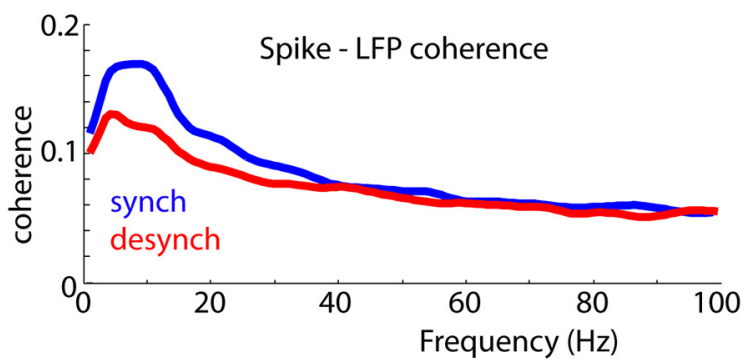

C

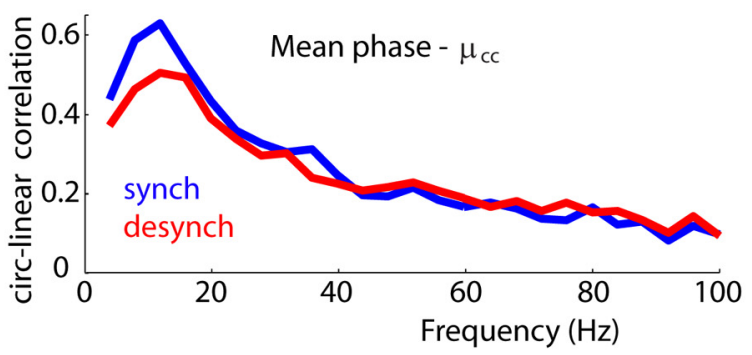

D
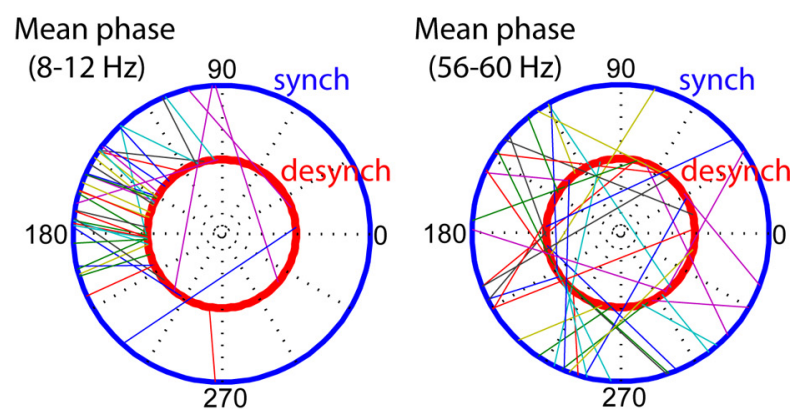

$\mathbf{E}$

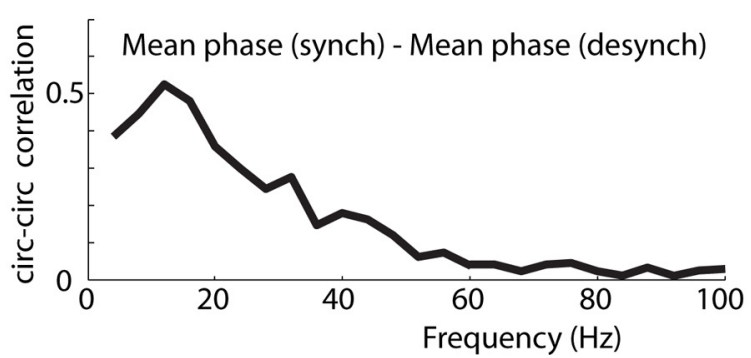

Figure 9. Spike timing with respect to MUA determines relation to LFP specifically at low frequencies. A, LFP power spectra in synchronized and desynchronized states. Note that lowfrequency (below $\sim 20 \mathrm{~Hz}$ ) power is higher in synchronized states, whereas gamma frequency $(50-80 \mathrm{~Hz})$ power is slightly higher in desynchronized states. $\boldsymbol{B}$, Spike-LFP coherence in both states. Note the prominent low-frequency peak in the synchronized state, which is smaller but still present in the desynchronized state. C, Circular-linear correlation coefficient quantifying the relationship between $\mu_{\mathrm{cc}}$ and preferred LFP phase (Fig. 8G) as a function of frequency band $(0-4 \mathrm{~Hz}, 4-8 \mathrm{~Hz}$, etc.). Note the low-frequency peak, which is larger in the synchronized state. D, Polar plots showing the relationship between preferred phases in synchronized and desynchronized states for LFP filtered in $8-12 \mathrm{~Hz}$ band (left) and $56-60 \mathrm{~Hz}$ band (right). Each line represents one neuron. The radial fan out of lines visible for the $8-12 \mathrm{~Hz}$ band indicates that ture of this fluctuation dependent on cortical state. How does this affect the information the neural population carries about the sustained tone frequency? To answer this, we divided the sustained epochs into overlapping $50 \mathrm{~ms}$ bins and separated these into groups based on the instantaneous population firing rate in each bin. For each group, we performed an informationtheoretic analysis in which the instantaneous firing rate vector was used to predict the sustained tone frequency by linear discriminant analysis (see Materials and Methods). As expected, bins of higher firing rate conveyed more information about the stimulus. However, for a given rate, we did not detect any further effect of cortical state (Fig. 10A). We thus conclude that although the desynchronized state leads to higher firing rates on average, activity packets in the synchronized state (where firing rate is instantaneously high) contain as much information as equivalent periods of the desynchronized state. Bins of high instantaneous rate also showed the strongest sequential organization, consistent with these bins corresponding to instantaneous occurrence of sequentially organized activity packets (Fig. 10B).

\section{Discussion}

Our main findings are summarized in Figure 11. Tone onsets reliably caused activity packets of 50-100 ms duration, and similar packets occurred sporadically during sustained tone responses and silence. These packets had a broadly preserved sequential structure across stimuli and across onset, sustained, and silent periods. However, both the firing rates and precise timing of the constituent neurons varied with tone frequency, and the additional spikes fired in response to sustained tones occurred within packets rather than spread evenly throughout the tone duration. In desynchronized states, fluctuations in population rate were reduced, but timing relationships between neurons were preserved. These data suggest a model where population activity is built from discrete packets whose content conveys information about the stimulus, occurring reliably at onset and irregularly at other times, with desynchronized activity consisting of multiple overlapping packets.

\section{Relation to single-cell analyses}

Recordings of single-neuron tone responses are typically interpreted as showing a large increase in firing rate in response to tone onset, and a smaller, more sharply tuned rate increase during the sustained period (Vaadia et al., 1982; Volkov and Galazjuk, 1991; Wang et al., 2005; Bartho et al., 2009). Our data, however, indicate that onset-evoked packets are typically not larger than those during the sustained period. There are some methodological differences between our work and other previous studies in awake animals, such as a focus on deep rather than superficial layers. Nevertheless, we suggest the apparent discrepancy to previous work reflects not a difference in cortical processing, but a view of cortical activity that cannot be seen from single-neuron analyses. The PSTH reflects not only a neuron's instantaneous firing rate but also the probability it will fire on any given trial. Reliable occurrence of packets at onset and irregular occurrence thereafter will thus cause the PSTH to be high at onset and lower during the sustained period.

$\leftarrow$

most neurons have the same preferred phase for synchronized and desynchronized states, which is not the case for higher frequencies (right). $\boldsymbol{E}$, Circular-circular correlation quantifying the similarity of preferred phase in synchronized and desynchronized states, as a function of frequency band. All plots except $\boldsymbol{D}$ show a mean over all rats. 
A

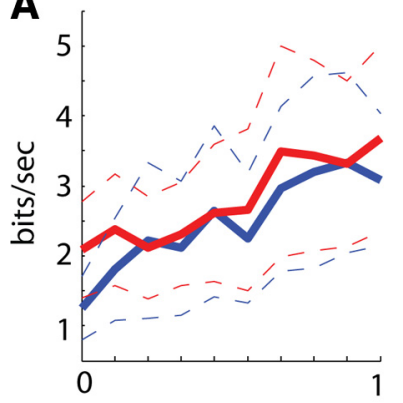

Firing rate interval (a.u.)

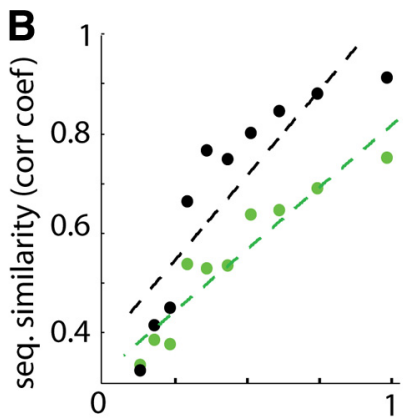

Normalized firing rate (a.u.)

Figure 10. Cortical state, population rate, and information coding. $\boldsymbol{A}$, Information coding depends on instantaneous population rate during the sustained period, but does not otherwise differ between states. Sustained-period responses were divided into 50 ms overlapping bins, and bins were divided into 10 groups corresponding to different population rates. Within each group, information about tone frequency was estimated for firing rate vectors occurring during synchronized (blue line) and desynchronized (red line) states (see Materials and Methods). Continuous lines show averages across all rats, and dashed lines show the \pm SEM. As expected, information content is higher during epochs of high rate, but for a given population rate there was no further effect of cortical state. $\boldsymbol{B}$, Periods of high instantaneous population rate also exhibit strongest sequential organization. For each of the 10 population rate groups, the stereotypy of sequential ordering among neurons was assessed by the correlation coefficient of $\mu_{c c}$ values to those measured in spontaneous activity (black dots; data from one representative experiment). To verify that the observed relationship was not just because sequential order can be more reliably evaluated from a larger number of spikes, we equalized the number of spikes in all groups by randomly removing spikes, and sequential order was recalculated (green dots).

\section{Synchronized state}
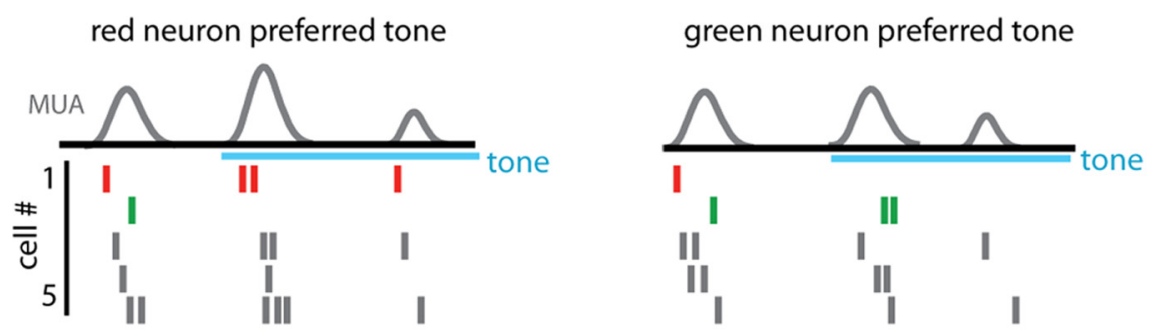

\section{Desynchronized state}
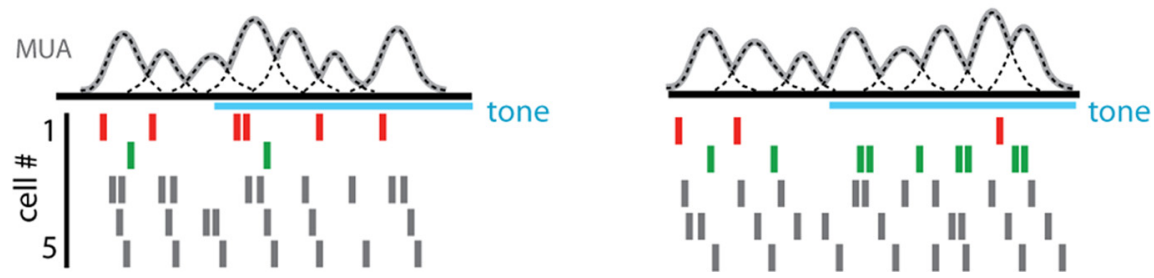

Figure 11. Model for auditory cortical population responses to sustained tones. In synchronized states (top), population activity consists of discrete packets separated by periods of global silence. Tone onset reliably induces an activity packet, but packets also occur sporadically throughout the sustained and spontaneous periods. Within each packet, neurons fire with a stereotyped sequential pattern. Presentation of a neuron's preferred tone causes it to fire at higher rates during activity packets, but not to fire outside packet periods. In desynchronized states (bottom), population activity does not show long periods of silence, but temporal relationships between neurons are similar to those in the synchronized state. This can be explained by a model in which many packets, individually similar to those seen in the synchronized state, are superimposed to produce a firing pattern that exhibits smaller fluctuations in global activity but retains a fine temporal structure.

Our results may explain a long-standing controversy regarding auditory cortical sustained responses during anesthesia. Sustained responses are always found in awake animals, but results under anesthesia have been inconsistent (e.g., Volkov and Galazjuk, 1991; deCharms and Merzenich, 1996; DeWeese et al.,

2003; Bartho et al., 2009). Our data suggest this could be explained by state differences between anesthetic conditions. For example, pentobarbital anesthesia (where sustained responses are typically not seen) produces a state in which spontaneous tivity packets are rare; under such conditions we suggest that tone onsets are capable of inducing an activity packet. Cony, urethane (where sustained responses can be seen) nization, as well as synchronized epochs with frequent spontanestimulus.

The present analyses made use of multisite population recordings, still a rarely used technique in sensory cortex. However, our results suggest a new interpretation of previous single neuron and LFP recordings. While LFP is often described in terms of oscillations, the dominant LFP pattern here was not rhythmic, but an irregular waveform mirroring the spontaneous activity packets. The LFP power spectrum correspondingly showed no narrowband peak, but a broadband signal $<20 \mathrm{~Hz}$, reciprocal to the $50-100 \mathrm{~ms}$ time scale of the activity packets. We found that and spike-field coherence in this frequency range was tuations in population rate caused by overlapping activity packets. Interestingly, studies in primate visual cortex have shown a imilar decrease in low-frequency LFP power, spike-field coherence, and interneuronal correlation when attention is directed into the receptive field of the recorded neuron (Fries et al., 2001; Cohen and Maunsell, 2009; Mitchell et al., 2009). We therefore hypothesize that population activity in primate visual cortex may show a homologous structure, with attention causing desynchronization of cortical activity on a localized basis (Harris and Thiele, 2011.)

\section{Possible mechanisms}

Several lines of evidence suggest that spontaneous firing packets are triggered by corticocortical rather than thalamocortical projections. First, voltage-sensitive dye imaging as well as recording studies in vivo and in vitro have shown that spontaneous activity often spreads as traveling waves over the cortical surface (Sanchez-Vives and McCormick, 2000; Ferezou et al., 2006; Luczak et al., 2007; Mohajerani et al., 2010), whose bilateral symmetry is disrupted in acallosal mice, consistent with its propagation through corticocortical synapses (Mohajerani et al., 2010). The timing of these spontaneous waves typically does not appear to be correlated to overt movements, and indeed they are suppressed during active behavior such as whisking (Ferezou et al., 2006). Second, while sensory responses are first seen in thalamorecipient layers, laminar analysis in vivo and in vitro indicates that spontaneous firing packets are first in the deep layers, which receive prolific corticocortical inputs (Sanchez-Vives and McCormick, 2000; Sakata and Harris, 2009). However, although different mechanisms may trigger spontaneous and sensory evoked firing packets, their spike patterns within a single layer are highly 
similar (MacLean et al., 2005; Luczak et al., 2009). Our data indicate that sustained-period packets are subject to similar constraints, suggesting that the same circuitry sculpts onset, spontaneous, and sustained firing packets.

Several studies suggest that the stereotyped temporal structure of cortical activity packets is imposed by the connectivity and physiology of the cortical microcircuit. Indeed, sequentially structured activity packets are seen in computational models of cortical circuits (Izhikevich et al., 2004; Izhikevich, 2006; Karmarkar and Buonomano, 2007; Buonomano and Maass, 2009; Fiete et al., 2010), in cortical slices (Mao et al., 2001; Buonomano, 2003; MacLean et al., 2005), as well as in response to sensory stimuli in vivo (Luczak et al., 2009). We found that population responses to different stimuli are subject to conserved spatiotemporal constraints, consistent with results in other modalities indicating conserved timing patterns in pairwise cross-correlograms (Jermakowicz et al., 2009). These results suggest that such activity packets represent the cortex's "impulse responses," with the precise spatiotemporal pattern produced dependent on the combination of input fibers active, subject to conserved constraints imposed by the circuitry. In this picture, activity in the desynchronized state, which is consistent with overlapping packets, could be viewed as the convolution of this impulse response with a continuous, weakly fluctuating input.

\section{Spontaneous activity as a gate for sensory responses}

Why should activity packets occur reliably at tone onset but sporadically during the sustained period? Tones produce large onset responses in subcortical auditory structures, which presumably evoke reliable cortical firing packets. In the sustained period, however, we hypothesize that ascending input is not sufficient to drive cortical firing, but that as during acoustic silence, top-down input causes sporadic packets of spontaneous activity in auditory cortex. When a tone is present, the precise activity pattern produced by auditory cortex in response to top-down input will depend on the ascending sensory input it receives. Top-down input thus opens a "gate" that lets through information about the external world. In the desynchronized state, these sporadic topdown barrages may be replaced by a more tonic top-down input pattern, analogous to a gate that is continuously open.

In this view, gating of sensory signals by spontaneous activity could be considered similar to a form of attention. Stimulus onsets are sufficient to open the gate by themselves, consistent with their "bottom-up" salience. Active sensory movements such as sniffs or saccades may also produce sufficient drive to open the gate, resulting in an activity packet similar to an onset response (Uchida et al., 2006; Melloni et al., 2009). In a passive animal exhibiting a synchronized state, however, the gate closes soon after stimulus onset, and only reopens sporadically during the sustained period, producing brief population bursts where information about external stimuli is broadcast from sensory cortex to the rest of the brain. Such an intermittent representation could have the benefit of interfering less with other cortical computations, as well as using less energy. In an alert or attentive animal exhibiting a desynchronized state, however, continuous topdown input allows an ongoing representation of the tone, corresponding to sustained "top-down" attention to this stimulus or to the sensory world in general. In primate visual cortex, lowfrequency power and coherence are locally suppressed by sustained attention (Fries et al., 2001; Mitchell et al., 2009), suggesting that desynchronization can be achieved in a retinotopically localized manner. We hypothesize that transient attention to a particular part of space may also be accompanied by a spa- tially localized "bump," corresponding to an increase in population firing rate, intracellular depolarization, and negative LFP deflection in a restricted area of cortex.

The picture that emerges from our results appears quite different from the common interpretation of spontaneous cortical activity as reflecting memory replay or consolidation (Buzsáki, 1989; Hoffman et al., 2007; O’Neill et al., 2010). The fact that spontaneous patterns occurring during sustained tone periods convey information about ongoing stimuli indicates that spontaneous activity does not always represent replay of prior experience, but rather can represent gated packets of information about ongoing external stimuli. We suggest that top-down input may play multiple roles in sensory cortex, with different patterns of descending input constituting different top-down "commands" that can trigger either the replay of previously stored experiences, or the representation of attended sensory stimuli.

\section{References}

Bartho P, Curto C, Luczak A, Marguet SL, Harris KD (2009) Population coding of tone stimuli in auditory cortex: dynamic rate vector analysis. Eur J Neurosci 30:1767-1778. CrossRef Medline

Battaglia FP, Benchenane K, Sirota A, Pennartz CM, Wiener SI (2011) The hippocampus: hub of brain network communication for memory. Trends Cogn Sci 15:310-318. Medline

Benucci A, Ringach DL, Carandini M (2009) Coding of stimulus sequences by population responses in visual cortex. Nat Neurosci 12:1317-1324. CrossRef Medline

Berens P (2009) CircStat: A MATLAB toolbox for circular statistics. J Stat Softw 31:1-21.

Breiman L (2001) Random forests. Machine Learn 45:5-32. CrossRef

Buonomano DV (2003) Timing of neural responses in cortical organotypic slices. Proc Natl Acad Sci U S A 100:4897-4902. CrossRef Medline

Buonomano DV, Maass W (2009) State-dependent computations: spatiotemporal processing in cortical networks. Nat Rev Neurosci 10:113-125. CrossRef Medline

Buzsáki G (1989) Two-stage model of memory trace formation: a role for 'noisy' brain states. Neuroscience 31:551-570. CrossRef Medline

Buzsáki G, Bickford RG, Ponomareff G, Thal LJ, Mandel R, Gage FH (1988) Nucleus basalis and thalamic control of neocortical activity in the freely moving rat. J Neurosci 8:4007-4026. Medline

Carr MF, Jadhav SP, Frank LM (2011) Hippocampal replay in the awake state: a potential substrate for memory consolidation and retrieval. Nat Neurosci 14:147-153. CrossRef Medline

Cohen MR, Maunsell JH (2009) Attention improves performance primarily by reducing interneuronal correlations. Nat Neurosci 12:1594-1600. CrossRef Medline

Constantinople CM, Bruno RM (2011) Effects and mechanisms of wakefulness on local cortical networks. Neuron 69:1061-1068. CrossRef Medline

Cortes C, Vapnik V (1995) Support-vector networks. Machine Learn 20: 273-297. CrossRef

Crochet S, Petersen CC (2006) Correlating whisker behavior with membrane potential in barrel cortex of awake mice. Nat Neurosci 9:608-610. CrossRef Medline

deCharms RC, Merzenich MM (1996) Primary cortical representation of sounds by the coordination of action- potential timing. Nature 381: 610-613. CrossRef Medline

deCharms RC, Blake DT, Merzenich MM (1998) Optimizing sound features for cortical neurons. Science 280:1439-1443. CrossRef Medline

de Kock CP, Bruno RM, Spors H, Sakmann B (2007) Layer- and cell-typespecific suprathreshold stimulus representation in rat primary somatosensory cortex. J Physiol 581:139-154. CrossRef Medline

DeWeese MR, Zador AM (2006) Non-Gaussian membrane potential dynamics imply sparse, synchronous activity in auditory cortex. J Neurosci 26:12206-12218. CrossRef Medline

DeWeese MR, Wehr M, Zador AM (2003) Binary spiking in auditory cortex. J Neurosci 23:7940-7949. Medline

Doron NN, Ledoux JE, Semple MN (2002) Redefining the tonotopic core of rat auditory cortex: Physiological evidence for a posterior field. J Comp Neurol 453:345-360. CrossRef Medline 
Dragoi G, Tonegawa S (2011) Preplay of future place cell sequences by hippocampal cellular assemblies. Nature 469:397-401. CrossRef Medline

Ferezou I, Bolea S, Petersen CC (2006) Visualizing the cortical representation of whisker touch: voltage-sensitive dye imaging in freely moving mice. Neuron 50:617-629. CrossRef Medline

Fiete IR, Senn W, Wang CZ, Hahnloser RH (2010) Spike-time-dependent plasticity and heterosynaptic competition organize networks to produce long scale-free sequences of neural activity. Neuron 65:563-576. CrossRef Medline

Fries P, Reynolds JH, Rorie AE, Desimone R (2001) Modulation of oscillatory neuronal synchronization by selective visual attention. Science 291: 1560-1563. CrossRef Medline

Greenberg DS, Houweling AR, Kerr JN (2008) Population imaging of ongoing neuronal activity in the visual cortex of awake rats. Nat Neurosci 11:749-751. CrossRef Medline

Han F, Caporale N, Dan Y (2008) Reverberation of recent visual experience in spontaneous cortical waves. Neuron 60:321-327. CrossRef Medline

Harris KD, Thiele A (2011) Cortical state and attention. Nat Rev Neurosci 12:509-523. CrossRef Medline

Hoffman KL, McNaughton BL (2002) Coordinated reactivation of distributed memory traces in primate neocortex. Science 297:2070-2073. CrossRef Medline

Hoffman KL, Battaglia FP, Harris K, MacLean JN, Marshall L, Mehta MR (2007) The upshot of up states in the neocortex: from slow oscillations to memory formation. J Neurosci 27:11838-11841. CrossRef Medline

Izhikevich EM (2006) Polychronization: computation with spikes. Neural Comput 18:245-282. CrossRef Medline

Izhikevich EM, Gally JA, Edelman GM (2004) Spike-timing dynamics of neuronal groups. Cereb Cortex 14:933-944. CrossRef Medline

Jermakowicz WJ, Chen X, Khaytin I, Bonds AB, Casagrande VA (2009) Relationship between spontaneous and evoked spike-time correlations in primate visual cortex. J Neurophysiol 101:2279-2289. Medline

Ji D, Wilson MA (2007) Coordinated memory replay in the visual cortex and hippocampus during sleep. Nat Neurosci 10:100-107. CrossRef Medline

Karmarkar UR, Buonomano DV (2007) Timing in the absence of clocks: encoding time in neural network states. Neuron 53:427-438. CrossRef Medline

Kenet T, Bibitchkov D, Tsodyks M, Grinvald A, Arieli A (2003) Spontaneously emerging cortical representations of visual attributes. Nature 425 : 954-956. CrossRef Medline

Luczak A, Bartho P, Marguet SL, Buzsáki G, Harris KD (2007) Sequential structure of neocortical spontaneous activity in vivo. Proc Natl Acad Sci U S A 104:347-352. CrossRef Medline

Luczak A, Barthó P, Harris KD (2009) Spontaneous events outline the realm of possible sensory responses in neocortical populations. Neuron 62:413-425. CrossRef Medline

MacLean JN, Watson BO, Aaron GB, Yuste R (2005) Internal dynamics determine the cortical response to thalamic stimulation. Neuron 48:811-823. CrossRef Medline

Mao BQ, Hamzei-Sichani F, Aronov D, Froemke RC, Yuste R (2001) Dynamics of spontaneous activity in neocortical slices. Neuron 32: 883-898. CrossRef Medline

Melloni L, Schwiedrzik CM, Rodriguez E, Singer W (2009) (Micro)saccades, corollary activity and cortical oscillations. Trends Cogn Sci 13:239-245. CrossRef Medline

Mitchell JF, Sundberg KA, Reynolds JH (2009) Spatial attention decorrelates intrinsic activity fluctuations in macaque area V4. Neuron 63: 879-888. CrossRef Medline

Mohajerani MH, McVea DA, Fingas M, Murphy TH (2010) Mirrored bilateral slow-wave cortical activity within local circuits revealed by fast bihemispheric voltage-sensitive dye imaging in anesthetized and awake mice. J Neurosci 30:3745-3751. CrossRef Medline

Moshitch D, Las L, Ulanovsky N, Bar-Yosef O, Nelken I (2006) Responses of neurons in primary auditory cortex (A1) to pure tones in the halothaneanesthetized cat. J Neurophysiol 95:3756-3769. CrossRef Medline

Narayanan NS, Kimchi EY, Laubach M (2005) Redundancy and synergy of neuronal ensembles in motor cortex. J Neurosci 25:4207-4216. CrossRef Medline

Nelken I, Chechik G, Mrsic-Flogel TD, King AJ, Schnupp JW (2005) Encoding stimulus information by spike numbers and mean response time in primary auditory cortex. J Comput Neurosci 19:199-221. CrossRef Medline

Niell CM, Stryker MP (2010) Modulation of visual responses by behavioral state in mouse visual cortex. Neuron 65:472-479. CrossRef Medline

Oh J, Laubach M, Luczak A (2003) Estimating neuronal variable importance with random forest. In: Proceedings of the IEEE 29th Annual Northeast Bioengineering Conference, pp 33-34 Newark, NJ: New Jersey Institute of Technology.

Okun M, Naim A, Lampl I (2010) The subthreshold relation between cortical local field potential and neuronal firing unveiled by intracellular recordings in awake rats. J Neurosci 30:4440-4448. CrossRef Medline

O'Neill J, Pleydell-Bouverie B, Dupret D, Csicsvari J (2010) Play it again: reactivation of waking experience and memory. Trends Neurosci 33:220-229. CrossRef Medline

Panzeri S, Petersen RS, Schultz SR, Lebedev M, Diamond ME (2001) The role of spike timing in the coding of stimulus location in rat somatosensory cortex. Neuron 29:769-777. CrossRef Medline

Poulet JF, Petersen CC (2008) Internal brain state regulates membrane potential synchrony in barrel cortex of behaving mice. Nature 454:881-885. CrossRef Medline

Qin L, Chimoto S, Sakai M, Wang J, Sato Y (2007) Comparison between offset and onset responses of primary auditory cortex ON-OFF neurons in awake cats. J Neurophysiol 97:3421-3431. CrossRef Medline

Reich DS, Mechler F, Victor JD (2001) Temporal coding of contrast in primary visual cortex: when, what, and why. J Neurophysiol 85: 1039-1050. Medline

Renart A, de la Rocha J, Bartho P, Hollender L, Parga N, Reyes A, Harris KD (2010) The asynchronous state in cortical circuits. Science 327:587-590. CrossRef Medline

Richmond BJ, Optican LM, Podell M, Spitzer H (1987) Temporal encoding of two-dimensional patterns by single units in primate inferior temporal cortex. I. Response characteristics. J Neurophysiol 57:132-146. Medline

Richmond BJ, Optican LM, Spitzer H (1990) Temporal encoding of twodimensional patterns by single units in primate primary visual cortex. I. Stimulus-response relations. J Neurophysiol 64:351-369. Medline

Rutkowski RG, Miasnikov AA, Weinberger NM (2003) Characterisation of multiple physiological fields within the anatomical core of rat auditory cortex. Hear Res 181:116-130. CrossRef Medline

Sakata S, Harris KD (2009) Laminar structure of spontaneous and sensoryevoked population activity in auditory cortex. Neuron 64:404-418. CrossRef Medline

Sally SL, Kelly JB (1988) Organization of auditory cortex in the albino rat: sound frequency. J Neurophysiol 59:1627-1638. Medline

Sanchez-Vives MV, McCormick DA (2000) Cellular and network mechanisms of rhythmic recurrent activity in neocortex. Nat Neurosci 3:10271034. CrossRef Medline

Shusterman R, Smear MC, Koulakov AA, Rinberg D (2011) Precise olfactory responses tile the sniff cycle. Nat Neurosci 14:1039-1044. CrossRef Medline

Takeuchi D, Hirabayashi T, Tamura K, Miyashita Y (2011) Reversal of interlaminar signal between sensory and memory processing in monkey temporal cortex. Science 331:1443-1447. CrossRef Medline

Uchida N, Kepecs A, Mainen ZF (2006) Seeing at a glance, smelling in a whiff: rapid forms of perceptual decision making. Nat Rev Neurosci 7:485-491. CrossRef Medline

Vaadia E, Gottlieb Y, Abeles M (1982) Single-unit activity related to sensorimotor association in auditory cortex of a monkey. J Neurophysiol 48: 1201-1213. Medline

Volkov IO, Galazjuk AV (1991) Formation of spike response to sound tones in cat auditory cortex neurons: interaction of excitatory and inhibitory effects. Neuroscience 43:307-321. CrossRef Medline

Vyazovskiy VV, Olcese U, Hanlon EC, Nir Y, Cirelli C, Tononi G (2011) Local sleep in awake rats. Nature 472:443-447. CrossRef Medline

Wang X, Lu T, Snider RK, Liang L (2005) Sustained firing in auditory cortex evoked by preferred stimuli. Nature 435:341-346. CrossRef Medline 